Editorial

\title{
Global Leprosy Status in 2020: Still Losing Touch
}

Carlos Franco-Paredes, ${ }^{1,2}{ }_{M D}, M P H$, Griselda Montes de Oca Sanchez, ${ }^{3} M D$, Cassandra White, ${ }^{4} P h D$

Leprosy, also known as Hansen's Disease, is one of the oldest diseases that has afflicted humanity. It has been described in ancient Egyptian and Indian texts dating 600 BCE. ${ }^{1}$ Additionally, the molecular fingerprint of Mycobacterium leprae - the major aetiologic agent of leprosy - has been identified in bioarchaeological records. ${ }^{1,2}$ Recent comparative genomic and phylogeographic analysis of Mycobacterium leprae has linked its molecular epidemiology to social historical events, suggesting that the global spread of leprosy is associated with migration of humans. ${ }^{2}$ For example, leprosy arrived to the Americas with West African populations entering the New World via the transatlantic slave trade. Subsequent waves of European migration into the Americas were responsible for later introductions of Mycobacterium leprae. ${ }^{2}$ In Europe, improvements in social conditions in the second half of the $16^{\text {th }}$ Century led to a substantial reduction of leprosy as genomic comparisons of ancient and modern strains of Mycobacterium leprae demonstrate remarkable similarity. ${ }^{2}$ Today, the clustering of cases of leprosy mainly occurs among individuals living in resource-poor settings with favourable ecological niches for Mycobacterium leprae to thrive. ${ }^{3}$

In 1954, the French philanthropist, Raoul Follereau, established World Leprosy Day to be observed on the last Sunday of January. ${ }^{4}$ In India, World Leprosy Day is observed on 30 January - coinciding with the anniversary of Mahatma Gandhi's death on the same day in 1948-in acknowledgement of Gandhi's efforts to destigmatise the disease and improve the lives of people living with it. ${ }^{4}$ Since its inception, the focus of World Leprosy Day has been on the promotion of activities to raise awareness of the disease and to reduce stigma, discrimination and the resulting social isolation. ${ }^{4}$ Despite these and other efforts to reduce the burden of leprosy, it remains a much neglected infectious disease among marginalised populations. ${ }^{5,6} \mathrm{By}$ 1985, the global prevalence of leprosy was 5.2 million cases (identified in 122 countries). As a result and with the advent of multidrug therapy (MDT) to treat leprosy, the
World Health Assembly in 1991 approved a resolution to eliminate leprosy by the year $2000 .{ }^{1}$ The indicator chosen to measure this goal was defined as a worldwide prevalence of $<1$ case per 10,000 population by the year $2000 .{ }^{5}$

Although leprosy is no longer considered a public health risk, according to the World Health Organization (WHO), new cases reached 600,000 in $2000 .{ }^{6}$ By 2005 , the number of new cases has remained relatively stable with around 200,000 cases reported annually. ${ }^{6}$ Detecting new cases is considered a marker of active and ongoing transmission ${ }^{6}$ of Mycobacterium leprae or Mycobacterium lepromatosisthe 2 mycobacterial species responsible for causing leprosy. ${ }^{1}$ This year's commemoration of World Leprosy Day on 26 January marks the $20^{\text {th }}$ anniversary of the elimination of leprosy as a public health concern. However, since "elimination" was reached on a global scale in 2000, at least 4 million new cases of leprosy have been identified. ${ }^{6}$ Many of these cases have been termed the "missing millions". They include people living with leprosy (including those not yet identified by national statistics), ${ }^{6,7}$ individuals with multibacillary forms of leprosy that have been partially treated with a WHO-recommended MDT regimen (but who remain with viable bacilli at the completion of therapy) ${ }^{8}$ and individuals who have completed MDT (but who suffer from late-onset leprosy reactions). ${ }^{9,10}$ Some regions and countries where leprosy had been declared "eliminated" in the past are now seeing a resurgence of the disease. ${ }^{11,12}$ Furthermore, many who have completed MDT are left with permanent neurological sequelae that result in deformities, dysfunction, limb loss, blindness and stigma. ${ }^{1,10}$

Leprosy is a chronic mycobacterial infection caused by Mycobacterium leprae and Mycobacterium lepromatosis that affects the peripheral nerve, skin and nasopharyngeal mucosa. ${ }^{1,13}$ The infection produces neurological dysfunction by directly infecting Schwann cells of the peripheral nerve leading to demyelination, neuritis and long-term sequelae in the majority of patients. ${ }^{1}$ Additionally, cutaneous and mucosal involvement occur due to infection of histiocytes and keratinocytes. Nerve injury leads to disabilities

\footnotetext{
${ }^{1}$ Division of Infectious Diseases, Department of Medicine, University of Colorado Anschutz Medical Center, United States of America

${ }^{2}$ Instituto Nacional de Salud, Hospital Infantil de México, Mexico

${ }^{3}$ Servicio de Dermatologia, Hospital General de México, Mexico

${ }^{4}$ Department of Anthropology, Georgia State University, United States of America

Address for Correspondence: Dr Carlos Franco-Paredes, Division of Infectious Diseases, Department of Medicine, University of Colorado Anschutz Medical Center, Aurora, Colorado 80045, United States of America.

Email: carlos.franco.paredes@gmail.com
} 
and deformities, making this disease destructive and stigmatising. ${ }^{9}$ Many patients - particularly those suffering from any of the multibacillary forms of the disease - develop acute inflammatory episodes called leprosy reactions, which in turn, produce worsening nerve damage. ${ }^{1,9}$ Many newly diagnosed cases have already developed grade I ( $40 \%)$ or grade II disability (28\%); ${ }^{5}$ many occur in children ${ }^{6}$ and most cases are multibacillary forms of leprosy. ${ }^{5}$ The "elimination" mantra of leprosy has already created significant confusion, particularly when the actual epidemiology of leprosy appears to be different from the number of official cases reported; and its mode of transmission remains to be fully elucidated. The incidence of leprosy in some endemic settings has remained stable over the last 3 decades (with the number of cases being 17 times higher than those reported officially). ${ }^{7}$ In summary, leprosy remains a public health concern in many settings where official reports do not capture the high levels of suffering and social injury of patients living with the disease $\cdot{ }^{14}$ Additionally, the epidemiology and burden of disease associated with Mycobacterium lepromatosis and its role in the severity of leprosy reactions need to be defined.

This World Leprosy Day, we celebrate the reduced overall prevalence of leprosy, the humility of individuals and dedication of philanthropic institutions that are involved in lessening the global effects of this disease. This day also provides an opportunity to perform a reassessment of leprosy as a remediable injustice that goes beyond the widespread deployment of MDT. The focus of the elimination campaign, which is on the employment of biomedical interventions, has proven to be insufficient. The indicators to define success in leprosy control, which have moved from addressing the prevalence of the disease to reducing its incidence, and more recently to preventing disability, offer incomplete approaches to this complex disease.

What are the reasons for the persistence of leprosy despite the widespread deployment of MDT to endemic areas? The continuous transmission of leprosy in many resource-poor settings indicates that many people in the world have limited access to good education, sanitation, health and social services and are affected by forced migration. ${ }^{2,3}$ Leprosy does not occur in an ecological and societal vacuum. Thus, a vertical approach to control or eliminate the disease is insufficient. Leprosy is one of many neglected diseasesincluding tuberculosis, lymphatic filariasis, Chagas disease and other tropical diseases - that is related to poverty and maldistribution of wealth. The global leprosy control programme has been successful in delivering MDT but leprosy is too complex for a simple elimination model. We believe that attaining a leprosy-free world requires social autopsies, which are assessments to highlight the importance of social and non-biological forces contributing to the continuous occurrence of leprosy and its sequelae ("beyond the numbers").This approach will help in the implementation of comprehensive horizontal interventions which invest in human and social capital and the overall well-being of affected individuals and communities. Additionally, optimal strengthening of healthcare systems and fostering of health equity are necessary to reduce the current unfair social arrangements that contribute to the persistent occurrence of leprosy and other neglected infectious diseases. Leprosy is an ancient disease that offers an opportunity to learn about its ecological expression of social inequalities, historical power of stigma and the endurance of the human spirit.

\section{REFERENCES}

1. White C, Franco-Paredes C. Leprosy in the $21^{\text {st }}$ century. Clin Microb Rev 2015;28:80-94.

2. Monot M, Honore N, Garnier T, Araoz R, Coppee JY, Jacroix C, et al. On the origin of leprosy. Science 2005;308:1040-2.

3. Kerr-Pontes LRS, Barreto ML, Evangelist CMN, Rodrigues LC, Heukelbach J, Feldmeier H. Socioeconomic, environmental, and behavioural risk factors for leprosy in Northeast Brazil: results of a case-control study. Int J Epidemiol 2006;35:994-1000.

4. Centers for Diseases Control and Prevention. World Leprosy Day: bust the myths, learn the facts. Available at: https://www.cdc.gov/features/ world-leprosy-day/index.html. Accessed on 30 November 2019.

5. World Health Organization. Global leprosy update, 2018. Moving towards a leprosy free world. Wkly Epidemiol Rec 2019;94:389-412.

6. Smith WC, van Brakel W, Gillis T, Saunderson P, Richardus JH. The missing millions: a threat to the elimination of leprosy. PLoS Negl Trop Dis 2015;9:e0003658.

7. Pedrosa Vl, Dias LC, Galban E, Leturiondo A, Palheta J Jr, Santos M, et al. Leprosy among schoolchildren in the Amazon region: a cross-sectional study of active search and possible source of infection by contact tracing. PLoS Negl Trop Dis 2018;12:e0006261.

8. Guerrero MI, Muvdi S, León CI. Relapses in multibacillary leprosy patients: a retrospective cohort of 11 years in Colombia. Lepr Rev 2012;83:247-60.

9. Jacob J, Kozarsky P, Dismukes R, Franco-Paredes C. Five-year experience with type 1 and type 2 reactions in Hansen's disease at a US Travel Clinic. Am J Trop Med Hygiene 2008;79:452-4.

10. White C. Leprosy and stigma in the context of international migration. Lepr Rev 2011;82:147-54.

11. Thangaraju P, Venkatesan S, Selvan TT, Sivshanmugam E, Ali MS. The resurgence of leprosy in India: findings from a survey assessing medical professionals' knowledge and preparedness. Educ Health Prof 2018;1:24-7.

12. Parajuli S, Pokhrel DB, Baskota R, Pouydal A, Paudel U. Challenges of sustaining elimination status of leprosy in Nepal. J Inst Med (Nepal) 2019;41:59-61.

13. Tan T. Leprosy. Ann Acad Med Singapore 1981;10:84-90.

14. Scollard DM. Treatment gets better, but leprosy remains a global problem. Ann Acad Med Singapore 2008;37:1-2. 